\title{
Experiência do trabalho de uma equipe de Enfermagem na imunização contra a
}

\section{Covid-19 pelo modelo drive-thru}

\author{
Experience of the work of a Nursing team in immunization against Covid-19 using the drive-thru \\ model
}
Experiencia del trabajo de un equipo de Enfermería en inmunización contra Covid-19 por el modelo drive-thru

\section{Resumo}

Objetivo: descrever a experiência do trabalho de uma equipe de Enfermagem na linha de frente da imunização em massa da população contra a COVID-19 pelo modelo drive-thru e os desafios enfrentados. Método: estudo descritivo do tipo relato de experiência sobre a atuação da equipe de Enfermagem, no período de duas semanas, na imunização contra a Covid-19 pelo modelo drive-thru em um estacionamento de um shopping de Fortaleza, Ceará, Brasil, em fevereiro de 2021. Resultados: o local de vacinação foi estruturado em logística adequada para atender ao quantitativo de até 400 veículos sob prévio agendamento via aplicativo, com imunização distribuída pela equipe previamente imunizada e treinada. O processo de trabalho de Enfermagem no manejo, manutenção, administração e descarte dos imunobiológicos seguiu a Rede de Frio do Programa Nacional de Imunização e os desafios enfrentados estavam relacionados aos insumos insuficientes, desinformação da população e logística que desfavorecia a outros tipos de veículos de transporte. Conclusão: A equipe de Enfermagem tem uma importante contribuição na operacionalização da Campanha Nacional de Imunização contra Covid-19, contudo, ressalta-se a necessidade de estratégias para a gestão local suprir as falhas na logística de fornecimento das vacinas, estratégias de viabilização do acesso à população que não possui automóvel e de combate às fake news.

Palavras-chave: Covid-19; Vacinação em massa; Enfermagem.

\begin{abstract}
Objective: to describe an experience of the work of a nursing team in the front line of mass immunization of the population against a COVID-19 by the drive-thru model and the challenges faced. Method: a descriptive study of the experience report type on the performance of the nursing team, over a two-week period, in the immunization against Covid-19 using the drive-thru model in a parking lot in a shopping center in Fortaleza, Ceará, Brazil, in February of 2021. Results: the vaccination site was structured in adequate logistics to meet the quantity of up to 400 vehicles under prior scheduling via the app, with immunization distributed by the previously immunized and trained team. The
\end{abstract}


nursing work process in the handling, maintenance, administration and disposal of immunobiologicals followed the Cold Chain of the National Immunization Program and the challenges faced were related to insufficient inputs, population misinformation and logistics that disfavored other types of transport vehicles. Conclusion: The Nursing team has an important contribution in the operationalization of the National Immunization Campaign against Covid19, however, it emphasizes the need for a strategy for local management to address the flaws in the logistics of supplying vaccines, strategies to enable access people who do not own a car and fight false news.

Keywords: Covid-19; Mass vaccination; Nursing.

\section{Resumen}

Objetivo: describir una experiencia del trabajo de un equipo de enfermería en la primera línea de inmunización masiva de la población contra un COVID-19 por el modelo drive-thru y los desafíos enfrentados. Método: estudio descriptivo del tipo informe de experiencia sobre el desempeño del equipo de enfermería, durante un período de dos semanas, en la inmunización contra Covid-19 utilizando el modelo drive-thru en un estacionamiento de un centro comercial de Fortaleza, Ceará, Brasil, en febrero de 2021. Resultados: el sitio de vacunación se estructuró en logística adecuada para atender la cantidad de hasta 400 vehículos bajo programación previa a través de la aplicación, con inmunización distribuida por el equipo previamente inmunizado y capacitado. El proceso de trabajo de enfermería en el manejo, mantenimiento, administración y disposición de inmunobiológicos siguió la Cadena de Frío del Programa Nacional de Inmunizaciones y los desafíos enfrentados se relacionaron con insumos insuficientes, desinformación poblacional y logística que desfavoreció a otro tipo de vehículos de transporte. Conclusión: El equipo de Enfermería tiene un aporte importante en la operacionalización de la Campaña Nacional de Inmunización contra Covid-19, sin embargo, enfatiza la necesidad de una estrategia de gestión local para abordar las fallas en la logística de suministro de vacunas, estrategias que permitan el acceso de las personas. que no tienen coche y luchan contra las noticias falsas.

Palabras clave: Covid-19; Vacunación masiva; Enfermería.

\section{Introdução}

Desde que declarada a Covid-19 como pandemia em 11 de março de 2020, a doença causada pelo novo coronavírus SARS-CoV-2 segue em curso com variações nas curvas epidêmicas e surgimento de novas variantes afetando milhares de pessoas. O último relatório da World Health Organization, de 16 de agosto de 2021, apontou 206.958.371 casos confirmados no mundo, com uma mortalidade de 4.357.179 de pessoas. O Brasil assumiu posição de terceiro lugar no ranking dos países de maior número de casos acumulados com 20.350 .142 e segundo lugar no número de mortes com 568.788 casos. A incidência de casos e óbitos neste momento, agosto de 2021, permanece no nível mais alto desde o início da pandemia (World Health Organization, 2021).

Os dados refletem a fragilidade dos sistemas de saúde do mundo pela alta demanda por atendimento de maior complexidade e densidade tecnológica, mesmo em países ricos e bem estruturados, e, ainda, a insuficiência das medidas de controle adotadas até o momento como a oferta de testes, uso de máscaras, isolamento social, quarentena aos expostos ou contaminados, evitando-se aglomerações (Couto, Barbieri \& Matos, 2021).

Mediante cenário mundial econômico caótico e colapso da saúde pública, agravados nos países em desenvolvimento como o Brasil, onde questões sociais, conflitos políticos e outras doenças infecciosas disputam recursos da saúde, iniciou-se, a busca pela produção e compra das vacinas como o meio mais eficaz de barrar a infecção pelo novo coronavírus (Anser et al., 2020).

A gravidade da pandemia impulsionou a Agência Nacional de Vigilância Sanitária (ANVISA) aprovar a imunização contra a Covid-19 em caráter emergencial com as vacinas produzidas até o momento. As vacinas de maior interesse para a população brasileira são de vírus inativado adsorvida Covid-19 (CoronaVac), parceria Sinovac e o Instituto Butantã elaborada no Brasil, vacinas com um vetor viral - um adenovírus atenuado (AAV), parceria do laboratório AstraZeneca, Universidade de Oxford e Fundação Osvaldo Cruz e Sputinik V ${ }^{\circledR}$ e vacinas produzidas de RNA mensageiro (RNAm) (Pfizer e Moderna) (Serpa et al, 2021).

A Campanha Nacional de Vacinação contra a Covid-19 teve início em janeiro de 2021, conforme diretrizes estabelecidas no Plano Nacional de Operacionalização da vacinação do Ministério da Saúde. As vacinas são ofertadas pelo 
Sistema Único de Saúde de forma gratuita à toda a população brasileira por meio do Programa Nacional de Imunizações, que é um dos maiores programas de vacinação do mundo, com reconhecimento internacional. Várias estratégias foram elaboradas para otimizar o processo de imunização, destacando-se vacinação em massa, capacitação dos profissionais de saúde, planejamento logístico de distribuição das vacinas, sistemas informatizados de acompanhamento e fases de vacinação por grupos prioritários (Ministério da Saúde, 2021).

Como estratégia para a vacinação em massa, destaca-se o modelo drive-thru que surgiu de experiências exitosas em campanhas de vacinação anteriores em diversos países, sendo considerado para este cenário de pandemia o meio mais rápido e seguro de imunizar o maior número de pessoas possível (Polo et al., 2020). O modelo tem sido apontado como mais eficiente do que a clínica tradicional (Kim, 2020), entretanto, pode apresentar vantagens e limitações variadas entre os países, tornandose necessário que a comunidade científica compartilhe as experiências de vacinação a fim de nortear a equipe de saúde e gestores mundiais para elaborar estratégias de melhorias em prol da população.

Quanto à equipe de Enfermagem, considerando que continuará sendo a maior categoria de profissionais da saúde na linha de frente à Covid-19 e, agora, com participação direta na operacionalização da vacinação em massa, faz-se necessário conhecer e compreender a atuação da Enfermagem nesse processo de trabalho, uma vez que o Conselho Federal de Enfermagem (2021) recomenda que as atividades de vacinação para Covid-19 funcionem como atividade extraordinária às rotinas de vacinação já existentes, com equipe capacitada para os procedimentos de manuseio, conservação, preparo, administração, registro e descarte dos resíduos advindos do material de vacinação, cujo dimensionamento de pessoal de enfermagem deve ocorrer de acordo com o porte do serviço e abrangência da população, em ambiente que possua locais de entrada e saída independentes para que garantam a integridade e proteção específica de profissionais e usuários dos serviços de saúde, além de todas as orientações quanto ao uso de equipamentos de proteção individual e medidas de distanciamento e organização local.

Neste sentido, o presente estudo se propõe a descrever a experiência do trabalho de uma equipe de Enfermagem na linha de frente da imunização em massa da população pelo modelo drive-thru e os desafios enfrentados.

\section{Metodologia}

\section{Desenho do estudo}

Trata-se de um estudo descritivo do tipo relato de experiência sobre a atuação da equipe de Enfermagem na campanha de imunização contra a COVID-19 pelo modelo drive-thru, com recorte temporal transversal. A narrativa científica elencada tem caráter de síntese provisória, está aberta para análise e discussão da comunidade acadêmica, assim como, o norteio para a produção de saberes novos e transversais (Daltro \& Faria, 2019).

\section{Período e local da experiência}

A experiência descrita se refere ao período de duas semanas vivenciadas por parte das pesquisadoras do manuscrito, em fevereiro de 2021, que ocorreu no estacionamento de um shopping center de grande porte, localizado no município de Fortaleza, Ceará, com logística sob planejamento da Secretaria da Saúde do Estado do Ceará.

\section{Participantes e critérios de seleção}

Participaram da estratégia de imunização equipes de profissionais de saúde, formadas por enfermeiros, técnicos de enfermagem, médicos, psicólogos, nutricionistas e fisioterapeutas, escalados por rodízio para comporem o total de uma equipe de 40 pessoas da escala diária para a operacionalização da fase 1 da Campanha Nacional de Vacinação contra a Covid-19, que 
iniciou com a vacinação do grupo prioritário de idosos na faixa etária maior ou igual a 75 anos, mediante cadastro no aplicativo Vacine Já do governo do Estado.

A Secretaria Municipal de Saúde, sob apoio da Secretaria da Saúde do Estado e a Escola de Saúde Pública do Ceará solicitou aos gestores dos serviços de saúde locais o recrutamento dos profissionais, que ocorreu por meios dos critérios disponibilidade de tempo e formação profissional na área da saúde, estabelecendo-se uma escala de trabalho para rodízio nos postos de vacinação. A equipe de Enfermagem, composta por oito profissionais, ficou responsável pelo manuseio, conservação, preparo, administração, registro e descarte dos imunobiológicos. Destaca-se que os profissionais de saúde recrutados foram previamente capacitados e vacinados com a primeira dose da vacina Coronavac antes de iniciarem os trabalhos no drive-thru, reduzindo o risco de exposição à população e aos próprios profissionais.

\section{Coleta e análise dos dados}

O conteúdo apresentado integra a observação da atuação de parte das autoras do manuscrito junto aos profissionais de Enfermagem atuantes na vacinação, cujos dados foram obtidos por meio das atividades desempenhadas pela equipe de Enfermagem desde a organização do processo de trabalho, manejo e manutenção das vacinas, segurança local, dos profissionais e usuários, procedimentos realizados, métodos adotados para manutenção da rede de frio quanto aos imunobiológicos e os desafios encontrados durante a implementação da estratégia de intervenção pelo modelo drive-thru.

A análise qualitativa e apresentação dos resultados se deu de forma descritiva da experiência vivenciada em três momentos: Logística e organização do processo de trabalho das equipes de vacinação no modelo drive-thru; Processo de trabalho de Enfermagem no manejo, manutenção, administração e descarte dos imunobiológicos; Desafios enfrentados pela equipe de vacinação.

Para melhor compreensão da logística local da experiência, foi realizada a reprodução por imagens por um profissional de design gráfico contratado para a reprodução gráfica do local da estratégia de imunização, que realizou por meio da modelagem base dos espaços no software Revit versão 2018 e da modelagem de itens pelo software SketchUp versão 2020. A aplicação da vegetação, das texturas e do preenchimento do espaço ocorreu pela humanização e render do software Lumion 10. Para o refinamento das imagens, a pós-produção, utilizou-se o Programa Photoshop CC versão 2019.

\section{Comitê de Ética}

A pesquisa não necessitou de apreciação do Comitê de Ética em Pesquisa, uma vez que se trata de um relato de experiência que não utilizou informações institucionais e de caracterização dos profissionais ou dos pacientes vacinados, entretanto, respeitou os aspectos éticos emanados pela resolução 466/2012 que diz respeito à pesquisa com seres humanos, preservando o anonimato de todos os envolvidos.

\section{Resultados}

\section{Logística e organização do processo de trabalho das equipes de vacinação no modelo drive-thru}

O estacionamento utilizado para o modelo drive-thru foi estruturado em tendas de suporte de segunda a sexta no período diurno e noturno, onde as equipes se alternavam conforme escala para as oito baias de vacinação, com dimensionamento mínimo de cinco profissionais das áreas da saúde e um vacinador (enfermeiro, técnico ou acadêmico de Enfermagem em supervisão). O local possuía capacidade para atendimento de 330 ou mais pacientes durante um único dia de serviço. Foram providenciadas entradas e saídas independentes para até 400 veículos organizados em filas para facilitar o acesso dos carros que transportavam os idosos ao local conforme ilustração na Figura1. 
Figura 1: Reprodução da área de acesso do estacionamento do shopping para a vacinação drive-thru contra Covid-19.

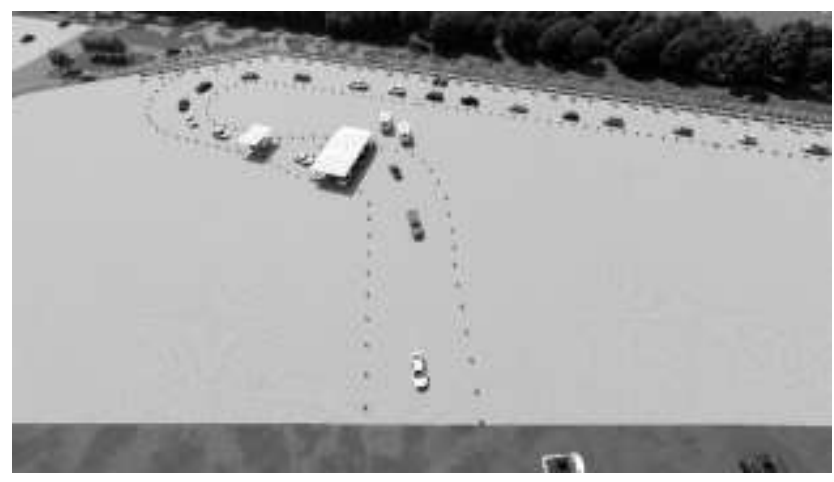

Fonte: Elaboração dos autores e profissional de desing gráfico.

Ao adentrarem no estacionamento, os carros eram direcionados para a triagem, onde uma equipe esclarecia dúvidas sobre a vacina, solicitava a apresentação da documentação necessária: carteira de identidade, comprovantes de endereço e de agendamento, carteira de vacina, caso não dispusesse, era ofertado e preenchido um cartão para o registro das doses da vacina, além de orientações para o agendamento prévio da segunda dose no site da prefeitura ou através do aplicativo Vacine Já.

Após esse processo de triagem, os integrantes da equipe sinalizavam para que os veículos se direcionassem às oito baias de vacinação, respeitando a ordem e o espaço sob a tenda. As baias de vacinação eram sinalizadas e separadas mecanicamente por cones de trânsito dispostos lado a lado com distância de um metro, mantendo a margem de segurança preconizada para evitar a exposição das vacinas e o risco de contaminação dos profissionais de Enfermagem que manipulavam a vacina. A estrutura é ilustrada nas Figuras 2 e 3.

Figura 2: Baias de vacinação e separação mecânica dos carros.

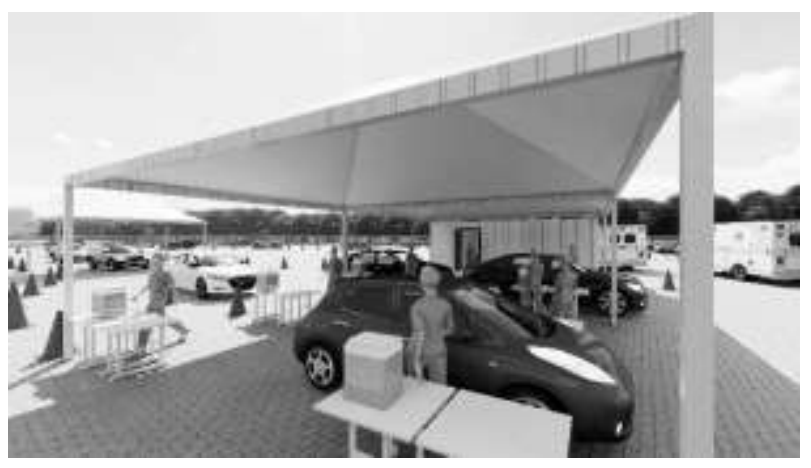

Fonte: Elaboração dos autores e profissional de desing gráfico.

Figura 3: Disposição dos carros e das caixas térmicas para imunização.

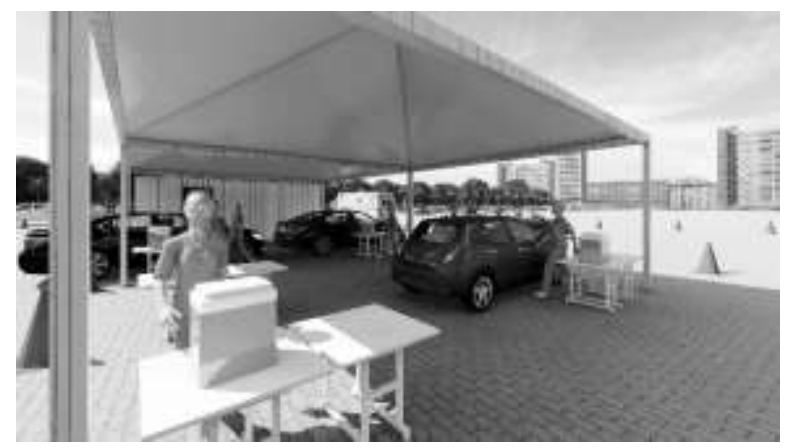

Fonte: Elaboração dos autores e profissional de desing gráfico. 
Em seguida, um membro da equipe se apresentava ao condutor do veículo, identificava a pessoa que iria se vacinar, a idade, o agendamento prévio e a documentação novamente, que entregava para um outro membro da equipe para dupla checagem das informações, inserindo-as no sistema de imunização do município de Fortaleza, por meio de um dispositivo tablet. Logo após era iniciado o preparo da dose e sua administração pelo profissional de enfermagem vacinador.

Nesta ação, houve a integração de acadêmicos de Enfermagem do ensino público e privado à estratégia de vacinação, os quais foram orientados a contribuírem inicialmente com o registro e cadastramento das pessoas, enquanto a vacina era preparada pelo profissional de Enfermagem da equipe. Após observarem muitos atendimentos, passaram a realizar o preparo das vacinas sob supervisão e administrá-las.

\section{Processo de trabalho de Enfermagem no manejo, manutenção, administração e descarte dos imunobiológicos}

A vacina ofertada foi a vacina covid-19 composta por partículas virais do vetor adenovírus recombinante do laboratório AstraZeneca (registro ANVISA 1.1063.0156), que possui esquema de duas doses com intervalo de 12 semanas. O imunobiológico possuía apresentação de solução injetável em embalagens de frascos-ampola de $5 \mathrm{ml}$, multidoses, contendo 10 doses para imunização, que ficavam acondicionados em caixas térmicas de poliuretano, com bobinas, em temperatura de $+2^{\circ} \mathrm{C}$ e $+8^{\circ} \mathrm{C}$ sob a tenda. A distribuição para as caixas térmicas ocorria em pouca quantidade havendo reposição conforme necessidade. Todo o recebimento, armazenamento, distribuição e transporte das vacinas ocorreu conforme orienta a Rede de Frio do Programa Nacional de Imunização.

No processo de trabalho, haviam duas enfermeiras responsáveis técnicas pela coordenação das equipes de vacinação nas baias e gestão de toda a documentação e protocolos de recebimento, distribuição, monitoramento de temperatura, gerenciamento de estoque e verificação da validade dos insumos, mantendo as medidas de segurança e conservação adequada dos imunobiológicos conforme Manual de Rede de Frio. O procedimento de preparo das doses era realizado pelo profissional de Enfermagem da equipe de cada baia, cuja dose recomendada era de 0,5ml, para administração via intramuscular, no músculo deltoide. Havia um carro de modelo trailer próximo às baias para armazenamento dos materiais e equipamentos de proteção individual (EPI) para os profissionais. Os demais profissionais da equipe realizavam a dupla checagem das informações dos usuários, de possíveis contraindicações e o registro da vacina nos cartões de vacinação e nos documentos de gestão interna.

Quando aos resíduos gerados pela vacinação, o material perfurocortante que incluía agulhas, seringas e frascos das vacinas eram descartados em coletores próprios com limite de capacidade até dois/terços do recipiente. Os EPIs eram descartados em lixo infectante e o material comum em lixo comum. As supervisoras técnicas estavam sempre reforçando sobre a higienização das mãos e das superfícies.

\section{Desafios enfrentados pela equipe de vacinação}

Durante a experiência de vacinação, perceberam-se idosos felizes pela oportunidade e alguns assustados, receosos e inseguros com medo de tomarem a vacina, até mesmo resistentes devido às informações divulgadas por amigos e redes sociais sobre reações que poderiam leva-los à morte pela imunização da vacina. Neste momento, fazia-se uma escuta qualificada, esclareciam-se as dúvidas pendentes, era reforçada a possibilidade de perda da integridade da vacina após preparada e não utilizada e, como ação de acolhimento e de humanização, utilizavam-se técnicas de relaxamento, notando-se que isso os acalmava. Aos que eram vacinados, o momento foi de emoção para profissionais, idosos e familiares que registraram com fotos, elogios e aplausos enaltecendo o Sistema único de saúde.

Observou-se, ainda, muitas dúvidas entre idosos e familiares, as principais foram sobre o laboratório, o intervalo das doses, os prazos, as reações da vacina e eficácia da vacina contra a nova variante do vírus SARS-CoV-2. Na oportunidade, a 
equipe de saúde fornecia orientações sobre a manutenção dos cuidados para evitar a contaminação e a transmissão da Covid19, fortalecendo as medidas de restrição social, o uso de máscaras, a higienização das mãos e dos objetos de uso pessoal e comercializados e, especialmente, os esclarecimentos sobre o aprazamento da segunda dose no cartão de vacinas e da necessidade de um novo agendamento.

No que diz respeito às vacinas, a quantidade total de doses adquiridas foi insuficiente para atender a demanda segundo o fluxo de gestão nas três esferas de governo repassada para o estado do Ceará e para o município de Fortaleza, tendo sido suspensa a vacinação após as duas semanas vivenciadas nessa experiência com retomada posterior em semanas subsequentes.

Outro fato interessante observado nessa experiência e que foi considerado um desafio foi que as pessoas que não dispunham de automóvel apareceram para vacinação a pé ou de transporte do tipo motocicleta, o que gerou constrangimento e aglomeração entre os carros, além do estresse para os motoristas e para a equipe de vacinação, pois interferiu na logística local e a estratégia não permitia a vacinação para pessoas com outros meios de transporte visando evitar o risco de quebrar as medidas de segurança estabelecidas pelas normativas técnicas do Ministério da saúde.

\section{Discussão}

O modelo de vacinação por drive-thru apresentou estrutura semelhante ao de vários países, com importantes benefícios como maior número de doses aplicadas, maior conforto e proteção à população, menor exposição ao vírus e baixo risco de transmissão de doenças para as equipes e público-alvo em comparação às unidades de saúde com instalações fechadas. A estratégia é oportuna para atender pessoas com problemas de mobilidade, geograficamente dispersas ou em restrição social. Em contrapartida, limita-se às condições meteorológicas, necessita de planejamento logístico, espaço amplo, adequado e controle do tráfego, a comunicação e o atendimento podem ser dificultados entre o profissional e as pessoas dentro do veículo caso apresentem algum mal-estar, além disso, há exposição da equipe de saúde ao monóxido de carbono e problemas de acessibilidade para as pessoas que não possuem carro (Asgary et al., 2020).

Vale ressaltar que o modelo de vacinação drive-thru utilizado e vivenciado na experiência generalizou o poder aquisitivo dos cidadãos como se a população em massa tivesse um veículo para se deslocar ao local de vacinação. Isso mostra que o modelo não beneficia pessoas de baixo perfil socioeconômico. Portanto, ressalta-se a importância de fortalecer estratégias que possam viabilizar a imunização em outros ambientes de acesso público, como escolas, quadras e ginásios esportivos, além das unidades de saúde.

No Brasil, a estratégia de vacinação em massa por drive-thru, foi adotada no Plano Nacional de Operacionalização da Vacinação contra covid-19, tendo como meta de imunizar de 60 a 70\% dos brasileiros para alcançar o controle da doença (Ministério da Saúde, 2021). A formação de grupos prioritários, baseada nos indicadores epidemiológicos, é uma estratégia importante e que assegura maior acessibilidade aos grupos vulneráveis. Destacando-se os idosos que representaram 53\% dos óbitos por covid-19 no Brasil em 2020, uma vez que a maioria das pessoas desta faixa etária já possuem outras comorbidades associadas, além da fragilidade do processo fisiológico do envelhecimento (Souto \& Kabad, 2020).

A experiência relatada na campanha de vacinação, no Ceará, corrobora com o estudo de Cunha et al. (2021) que relatou a atuação da enfermagem na campanha de vacinação contra a Covid-19 em um Centro Universitário em Belém do Pará e, ainda, permitiu observar que a Enfermagem participa ativamente e contribui com o planejamento e a gestão Logística da Rede de Frio, com vistas a promover a qualidade e a segurança no armazenamento no manuseio e na distribuição das vacinas (Ministério da Saúde, 2017). A atuação da Enfermagem vai desde o recebimento, verificação, triagem, armazenamento em temperatura adequada, preparo, administração de imunobiológicos, descarte correto dos resíduos, além da organização da estrutura de distribuição, treinamento da equipe pelo enfermeiro, registro de documentos para relatórios de produtividade e 
levantamento de indicadores de desempenho úteis a tomada de decisão e prática de auditoria interna e orientação da população (Ministério da Saúde, 2017; G. Oliveira et al., 2021).

Toda a equipe de enfermagem deve ser treinada e capacitada quanto às boas práticas de imunização, que compreendem: o acolhimento aos usuários do serviço, com empatia e presteza; a triagem do histórico de saúde e vacinal, verificação das indicações e contraindicações à vacinação, como pessoas com sintomas respiratórios; o controle rigoroso da Cadeia de Frio; a administração segura, em que o vacinador deve checar corretamente o paciente, a vacina, a dose, o preparo, a via de administração e o registro; a preparação de vacina em frascos-ampola multidose e monodose seguindo as recomendações de identificação dos frascos, aspiração das doses, utilização das agulhas e atenção aos prazos de validade; o descarte correto dos resíduos com materiais biológicos e perfurocortantes; o registro correto do imunizante administrado no documento vacinal; o uso dos equipamentos de proteção individual que incluem máscara cirúrgica, óculos ou protetor facial, avental e luvas, se necessário, somado ao distanciamento físico, etiqueta respiratória e lavagem frequente das mãos e descontaminação das superfícies; e a orientação e vigilância dos eventos adversos (Sociedade Brasileira de Pediatria, 2021).

Tendo em vista, os desafios enfrentados, o processo de imunização por meio de imunobiológicos produzidos no nosso país e imunobiológicos negociados e adquiridos com outros países gerou muitas dúvidas relacionadas a eficácia e às reações adversas, que foram fortalecidas por noticiários de casos de pessoas em outros países que se contaminaram mesmo tendo iniciado as doses das vacinas e por fake news de mídias televisivas e sociais sobre o vírus e suas variantes (Vasconcelos-Silva \& Castiel, 2020; Galhardi et al., 2021).

A hesitação da vacina contra a Covid-19 que consiste na relutância, indecisão ou recusa em se vacinar é um processo social complexo, que tem crescido em todo o mundo mesmo com os avanços de enfrentamento e controle da doença. As redes sociais e mídias tradicionais têm contribuído para disseminar polarizações políticas, teorias conspiratórias, movimento antivacina e preocupações relacionadas às vacinas, cujas informações incompletas têm alcance muito mais rápido do que os pareceres técnicos e científicos confiáveis, implicando, significativamente, em todo o processo de adesão às vacinas (B. Oliveira et al., 2021).

Vários são os desafios enfrentados em todo o país, os quais vão desde o processo lento de negociação das vacinas até a operacionalização delas em um país de grande área geográfica e densidade populacional, que enfrenta sérias iniquidades sociais, econômicas e culturais. O Brasil enfrenta problemas como a falta e o retardo de insumos suficientes para a produção em massa e, especialmente, as incompatibilidades políticas entre as instâncias de governo federal, estadual e municipal. Entretanto, o país possui uma grande vantagem pela existência do Programa Nacional de Imunização do Sistema Único de Saúde, que possui larga experiência, em torno de 46 anos, na dispensação de uma variedade de vacinas no país, que tem aumentado os esforços para superar dos desafios logísticos da dimensão geográfica (Guimarães et al, 2020).

O Brasil, no momento, sofre uma grave crise sanitária e política de intensa desvalorização da pesquisa científica que têm impacto direto no controle da doença, sendo necessário determinar uma comissão parlamentar de inquérito nacional (CPI) de Covid-19 pelo requerimento n. 1.371/21 para apurar as ações e omissões do governo federal no enfrentamento da pandemia por Covid-19 no Brasil e do colapso da saúde no estado do Amazonas (Senado Federal, 2021a), cujo relatório final identificou vários crimes envolvendo a epidemia com resultado em morte, devido ao negacionismo em relação ao vírus e às vacinas, à corrupção nas negociações para a compra de vacinas, às mortes provocadas pelo uso de tratamentos sem respaldo científico contra a covid-19, às infrações de medidas sanitárias preventivas e à prevacarização (Senado Federal, 2021b).

Neste panorama, a Enfermagem tem atuado intensivamente na linha de frente de combate à doença, tendo também, em contrapartida, muitos profissionais infectados e com suas vidas perdidas, outros desenvolvendo doenças mentais. O momento de pandemia foi oportuno para o reconhecimento da sociedade quanto a sua importância no controle da doença, que mais uma vez se faz essencial nesse período de imunização. A equipe de Enfermagem é responsável pela supervisão, 
administração das vacinas e monitoramento de toda a assistência de Enfermagem prestada à população com zelo, ética e competência técnica, além disso, possuem importante papel na orientação da população, tornando-se fundamentais para garantir o sucesso da campanha nacional de vacinação contra a Covid-19 (Castilho, 2021). A participação dos acadêmicos de Enfermagem agrega substancialmente à sua formação acadêmica, pois os estudantes aprendem sobre a composição dos imunobiológicos, correto armazenamento, normas de biossegurança, estratégias de triagem, administração de vacinas, fornecem apoio logístico e diminuem a demanda da equipe de enfermagem, tendo a oportunidade de uma experiência profissional única, em um momento histórico, além da sua contribuição social para a saúde da população (Botelho et al., 2021).

Portanto, compartilhar as vivências desse processo de trabalho durante a campanha de vacinação, destaca as potencialidades da Enfermagem em seu trabalho multidisciplinar na aplicação das ações e recomendações da Rede de Frio Nacional, evidencia as vantagens e desvantagens das ações inovadoras nos métodos de vacinação envolvendo o modelo drivethru, o agendamento online, subsidiando a operacionalização da campanha de vacinação em prol da qualificação da assistência à população e de todo o trabalho dos profissionais de saúde envolvidos.

\section{Limitações e implicações para a prática}

$\mathrm{O}$ estudo se limita por se tratar de um relato transversal com recorte temporal definido, que não permite avaliar a relação de causa e efeito, ademais se trata de um relato de experiência sob a perspectiva da equipe de enfermagem e um único local de vacinação.

O presente estudo traz contribuições para a produção do conhecimento de enfermagem na área temática, com descrição da experiência do processo de trabalho de Enfermagem na estratégia de imunização em massa pelo modelo drivethru, com reflexões importantes sobre o efeito das fake News, o desfavorecimento do modelo de imunização às pessoas de baixo perfil sociodemográfico, o impacto da desinformação e das irregularidades de governo, o que pode nortear ações e estratégias de gestores locais que otimizem a operacionalização da Campanha Nacional de imunização contra covid-19.

\section{Conclusão}

Com esta experiência, é notória a imprescindível contribuição da equipe de Enfermagem na operacionalização da Campanha Nacional de Imunização contra Covid-19 desde a supervisão, manutenção, preparo, administração das vacinas, descarte dos resíduos e orientação da população. O modelo de vacinação por drive-thru mostrou-se efetivo na otimização da imunização da população, ao mesmo tempo que, reduz o risco de transmissão do vírus e as aglomerações de idosos em filas de espera nas unidades básicas de saúde. Contudo, ressalta-se a necessidade dos gestores locais em discutir sobre as falhas na logística de fornecimento das vacinas, as estratégias de viabilização do acesso à população que não possui transporte e de combate às fake news. Recomenda-se que pesquisas posteriores investiguem o impacto da imunização em massa na diminuição dos casos de Covid-19, os problemas enfrentados para completar o esquema de vacinação com a segunda dose da vacina e o impacto que a intensa jornada de trabalho na imunização pode acarretar à vida dos profissionais de Enfermagem.

\section{Referências}

Anser, M. K., Yousaf, Z., Khan, M. A., Nassani, A. A., Alotaibi, S. M., Abro, M. M. Q., Vo, X. V. \& Zaman, K. (2020). Does comunica blediseases (including COVID-19) mayincrease global povertyrisk? A cloud onthehorizon. Environ Res. 187: e109668. 10.1016/j.envres.2020.109668.

Asgary, A., Valtchev, S. Z., Chen, M., Najafabadi, M. M. \& Wu, J. (2021). Artificial Intelligence Model of Drive-Through Vaccination Simulation. Int. J. Environ. Res. Public Health, 18 (268): e10268. 10.3390/ijerph18010268.

Botelho, J. L. S., Oliveira, M. D., Souza, N. A., Freitas, I. G. C., Ruas, R. F. B. \& Neta, A. I. O. (2021). Campanha de vacinação na Pandemia de SarsCov2: Relato de experiência. Revista Nursing, 24 (272): 5092-5094 10.36489/nursing.2021v24i272p5092-5097. 
Castilho, L. (2021). A Enfermagem como foco principal ao sucesso da vacinação contra a COVID-19. Revista nursing. (274):5344-5345. http://revistas.mpmcomunicacao.com.br/index.php/revistanursing/article/view/1319/1515.

Conselho Federal de Enfermagem. (2021). Medidas de prevenção e controle para profissionais na vacinação contra covid-19. http://www.cofen.gov.br/medidas-de-prevencao-e-controle-para-profissionais-na-vacinacao-contra-covid-19_84612.html

Couto, M. T., Barbieri, C. L. A. \& Matos, C. C. S. A. (2021). Considerations on COVID-19 impact on the individual-society relationship: from vaccine hesitancy to the clamor for a vaccine. Saúde Soc. 30(1): e200450. 10.1590/S0104-12902021200450

Cunha, A.G., Carvalho, D. P., Neri, D. T., Passinho, A. M. Leão, B. B. Castro, A. C. P., \& Furtado, A. R. D. (2021) Atuação da enfermagem na campanha de vacinação contra a COVID-19 em um Centro Universitário em Belém-PA. Research, Society and Development. 10 (8), e35310816835. http://dx.doi.org/10.33448/rsd-v10i8.16835

Daltro, R. M. \& Faria, A. M. (2019). Relato de experiência: Uma narrativa científica na pós-modernidade. Estud. pesqui. psicol. 19(1), 223-7. https://www.epublicacoes.uerj.br/index.php/revispsi/article/view/43015/29664.

Galhardi, C. P., Freire, N. P., Mynaio, M. C. S. \& Fagundes, M. C. M. (2020). Fato ou Fake? Uma análise da desinformação frente à pandemia da Covid-19 no Brasil. Ciência \& Saúde Coletiva, 25(2), 4201-4210. https://doi.org/10.1590/1413-812320202510.2.28922020.

Guimarães, R. (2020). Vacinas Anticovid: um Olhar da Saúde Coletiva. Ciência \& Saúde Coletiva. 25(9), 3579-3585. https://doi.org/10.1590/141381232020259.24542020 .

Kim, E. (2020). Repurposing COVID-19 Drive-Through Testing Centers for Mass Vaccination. Journal of Multidisciplinary Healthcare, $13: 1665-1667$. 10.214 7/JMDH.S279618

Ministério da Saúde. (2021). Plano Nacional de Operacionalização da vacinação contra COVID-19. Brasília-DF, (4a ed.), https://www.gov.br/saude/ptbr/media/pdf/2021/janeiro/29/PlanoVacinaoCovid_ed4_15fev21_cgpni_18h05.pdf

Ministério da Saúde. Secretaria de Vigilância em Saúde. Departamento de Vigilância das Doenças Transmissíveis. (2021). Manual de Rede de Frio do Programa Nacional de Imunizações. Secretaria de Vigilância em Saúde, Departamento de Vigilância das Doenças Transmissíveis. (5a ed.), Ministério da Saúde. https://portalarquivos2.saude.gov.br/images/pdf/2017/dezembro/15/rede_frio_2017_web_VF.pdf

Oliveira, B. L. C. A., Campos, M. A. G., Queiroz, R. C. S., Alves, M. T. S. S. B., Souza, B. F., Santos, A. M. \& Silva, A. A. M. (2021). Prevalence and factors associated with covid-19 vaccine hesitancy in Maranhão. Rev Saude Publica. 55: 12. http://dx.doi.org/10.11606/s1518-8787.2021055003417

Oliveira, G. C. A, Imperador, C., Ferreira, A. R. O., Oliveira, W. R., Camparoto, C. W., Jesus, W. A., \& Machado, M. F. (2021) Assistência de enfermagem no processo de imunização: revisão da Literatura. Brazilian Journal of Development, Curitiba. 7 (1), 7381-7395. https://doi.org/10.34117/bjdv7n1-499.

Polo, A., Schiavon C., Brancher, M., Cian, S., Zallot, C., Pupo, A., Rizzato, D. \& Cinquetti, S. (2021). Drive-through vaccinations prove successful in immunizing mountain communities against tick-borne encephalitis during the COVID-19 pandemic. Prev Med Hyg, 61:e497-E500. 10.15167/24214248/jpmh2020.61.4.1814.

Senado Federal. (2021a). CPI da COVID-19. Plano de trabalho 2021. https://cdn.oantagonista.net/uploads/2021/04/Plano-de-trabalho.docx-2.pdf.

Senado Federal. (2021b) Comissão Parlamentar de Inquérito da Pandemia. CPIPANDEMIA - CPI da Pandemia. Relatório Final http://estaticog1.globo.com/2021/10/20/cpidacovidrelatoriofinal.pdf?_ga=2.211787861.1140749082.1636823646-3196639742.1632858529

Serpa, F. S., Dortas-Junior, S.D., Guidacci, M. F. R. C., Sarinho, F. W., Silva, E. C., Rosario-Filho, N. A., \& Campos, R.A. (2021). Vacinas COVID-19 e imunobiológicos. Arq Asma Alerg Imunol. 5(2):126-34. http://dx.doi.org/10.5935/2526-5393.20210022

Sociedade Brasileira de Pediatria. (2021) Departamento Científico de Imunizações (2019-2021). Guia Prático de Atualização. Boas Práticas em Vacinação: Evitando Erros. (5). https://sbim.org.br/images/files/notas-tecnicas/23045c-gpa-boaspraticas_em_vacinacao-_evitandoerros.pdf

Souto, E. P. \& Kabad, J. (2020). Vaccine hesitancy and the challenges of dealing with the COVID-19 pandemic among older adults in Brazil. Rev. Bras. Geriatr. Gerontol, 23:5:e210032. http://dx.doi.org/10.1590/1981-22562020023.210032.

Vasconcellos-Silva, P. R. \& Castiel, L. D. (2020). COVID-19, as fakes news e o sono da razão comunicativa gerando monstros: a narrativa dos riscos e os riscos das narrativas. Cadernos de Saúde Pública. 2020, v. 36, n. 7

World Health Organization. (2021). Coronavirus (COVID-19) Dashboard. https://covid19.who.int/. 